\title{
SYAP1 expression associates with survival in triple negative breast cancer.
}

Shahan Mamoor, $\mathrm{MS}^{1}$

${ }^{1}$ shahanmamoor@gmail.com

East Islip, NY USA

We mined published microarray data (1) to understand the most significant gene expression differences in the tumors of triple negative breast cancer patients based on survival following treatment: dead or alive. We observed significant transcriptome-wide differential expression of synapse associated protein 1, encoded by SYAP1 when comparing the primary tumors of triple negative breast cancer patients dead or alive. Importantly, SYAP1 expression was correlated with overall survival in basal subtype breast cancer, a molecular subtype sharing significant overlap with triple negative breast cancer. SYAP1 may be of relevance as a biomarker or as a molecule of interest in understanding the etiology or progression of triple negative breast cancer.

Keywords: triple negative breast cancer, TNBC, systems biology of breast cancer, targeted therapeutics in breast cancer, SYAP1, synapse associated protein 1. 
Triple negative breast cancer is defined by lack of expression of the receptors for the hormones progesterone and estrogen (PR negative and ER negative), as well as for the human epidermal growth factor receptor 2 (HER2 negative) $(2,3)$. Though not completely identical, triple negative breast cancer shares significant overlap with the basal or basal-like molecular subtype of human breast cancer (3). TNBC is more frequently diagnosed in women of African descent (black women) for reasons not understood, and their disease course is similarly more aggressive than in white women with triple negative breast cancer $(2,3)$. To understand the transcriptional biology of triple negative breast cancer in an unbiased fashion and at the systems level, we mined published microarray data paired with patient survival data (1), performing comparative transcriptome analysis based on survival at time of data collection: dead or alive. We present here a finding from the results of this analysis.

\section{Methods}

We used dataset GSE142102 (1) for this differential gene expression analysis in conjunction with GEO2R. GSE142102 was generated using Affymetrix Human Gene 2.1 ST Array technology; in this analysis, we used data from $n=87$ tumors from TNBC patients that were dead at time of analysis and $n=123$ tumors from TNBC patients that were alive at time of analysis. The Benjamini and Hochberg method of p-value adjustment was used for ranking of differential expression but raw $p$-values were used to assess statistical significance of global differential expression. Log-transformation of data was auto-detected, and the NCBI generated category of platform annotation was used. A statistical test was performed to evaluate whether SYAP1 expression was significantly different between the primary tumors of TNBC patients that were dead or alive using a two-tailed t-test. For Kaplan-Meier survival analysis, we used the Kaplan-Meier plotter tool (4) for correlation of SYAP1 mRNA expression levels with overall survival (OS) in $n=296$ patients with basal subtype breast cancer.

\section{Results}

We performed comparative transcriptome analysis using published microarray data (1) to understand in an unbiased fashion the most significant gene expression differences in the primary tumors of patients with triple negative breast cancer based on survival outcomes.

SYAP1 is differentially expressed in triple negative breast cancer and negatively correlates with survival.

When comparing primary tumors of 87 live patients with triple negative breast cancer with that of 123 dead TNBC patients we identified synapse associated protein 1, SYAP1, as among the most significant transcriptional differences based on survival in human triple negative breast cancer. When ranking each of the transcripts whose expression was measured by microarray based on significance of difference in expression between the tumors of patients dead and alive, SYAP1 ranked 4 out of 53617 total transcripts, equivalent to $99.99 \%$ differential expression (Chart 1). SYAP1 differential expression in the tumors of patients based on survival outcomes was statistically significant (Figure $1 ; p=0.00007031$ ).

SYAP1 is expressed at significantly lower levels in the breast tumors of TNBC patients when comparing based on survival.

We obtained exact mRNA expression levels for SYAP1 from breast tumors of TNBC patients dead and alive to understand the magnitude and direction of SYAP1 expression change based on survival outcomes. SYAP1 was expressed at lower levels in tumors of TNBC patients alive as compared to that of 
TNBC patients dead (Figure 1). Decreased expression of SYAP1 in primary breast tumors was statistically significant (Figure 1: $p<0.0001$ ). SYAP1 was expressed at $0.33 \pm 1.05$ arbitrary units $(A U)$ in the primary tumors of TNBC patients dead, while it was expressed at $-0.23 \pm 0.89 \mathrm{AU}$ in the primary tumors of TNBC patients alive.

$\underline{\text { SYAP1 expression correlates with overall survival in basal subtype human breast cancer. }}$

We referenced a bioinformatics tool containing Kaplan-Meier survival data of human breast cancer patients to understand more thoroughly whether a relationship existed between SYAP1 primary tumor expression and patient survival in human breast cancer. We found that SYAP1 expression was correlated with overall survival in patients with basal or basal-like human breast cancer, a breast cancer molecular subtype which shares significant overlap with triple negative breast cancer (Figure 2; $\log$ rank $p$-value: 0.088 for overall survival, hazard ratio: $0.66(0.41-1.07)$ (Fig. 2)). SYAP1 mRNA levels were a positive prognostic indicator in basal subtype breast cancer patients. Median OS was, on average, markedly shorter for basal subtype patients with low tumor expression of SYAP1 as compared to basal subtype patients with high tumor expression of SYAP1.

Thus, we found through unbiased comparative transcriptome analysis that SYAP1 was among the genes whose expression was most significantly different in the primary tumors of triple negative breast cancer patients alive when compared to that of TNBC patients dead, that SYAP1 was expressed at significantly lower levels in the primary tumors of TNBC patients alive as compared to TNBC patients dead, and that SYAP1 expression was positively correlated with overall survival in the basal or basal-like molecular subtype.

\section{Discussion}

It is not understood why women of African descent (black women) are diagnosed with triple negative breast cancer more frequently than white women, and why this disease is more aggressive in black women. Deletion of the SYAP1 homologue in Drosophila (SAP47) results in deficits in associative memory and short-term plasticity (5); however, SYAP1 was cloned from a human liver cDNA library based on its down-regulation in hepatocellular carcinoma cells and named as such due to its homology to SAP47 (6). SYAP1 may be of relevance as a biomarker in identifying patients who are more likely to survive, perhaps as part of a panel of genes used for prognostic stratification, and the molecule itself may be important to the biology of triple negative breast cancer etiology or more likely its progression. Blind systems-level analyses, like this, can help shed light on the fundamental molecular characteristics of a disease whose racial propensities demand further explanation. 


\section{References}

1. Purrington, K.S., Knight III, J., Dyson, G., Ali-Fehmi, R., Schwartz, A.G., Boerner, J.L. and Bandyopadhyay, S., 2020. CLCA2 expression is associated with survival among African American women with triple negative breast cancer. PloS one, 15(4), p.e0231712.

2. Foulkes, W.D., Smith, I.E. and Reis-Filho, J.S., 2010. Triple-negative breast cancer. New England journal of medicine, 363(20), pp.1938-1948.

3. Dietze, E.C., Sistrunk, C., Miranda-Carboni, G., O'regan, R. and Seewaldt, V.L., 2015. Triple-negative breast cancer in African-American women: disparities versus biology. Nature Reviews Cancer, 15(4), pp.248-254.

4. Györffy, B., Lanczky, A., Eklund, A.C., Denkert, C., Budczies, J., Li, Q. and Szallasi, Z., 2010. An online survival analysis tool to rapidly assess the effect of 22,277 genes on breast cancer prognosis using microarray data of 1,809 patients. Breast cancer research and treatment, 123(3), pp.725-731.

5. Schmitt, D., Funk, N., Blum, R., Asan, E., Andersen, L., Rülicke, T., Sendtner, M. and Buchner, E., 2016. Initial characterization of a Syap1 knock-out mouse and distribution of Syap1 in mouse brain and cultured motoneurons. Histochemistry and cell biology, 146(4), pp.489-512.

6. Chang, Y.C., Yu, Y.L., Wang, N. and Xu, Y.H., 2001. Cloning and characterization of syap1, a down regulated gene in human hepatocellular carcinoma. Shi yan sheng wu xue bao, 34(4), pp.319-322. 


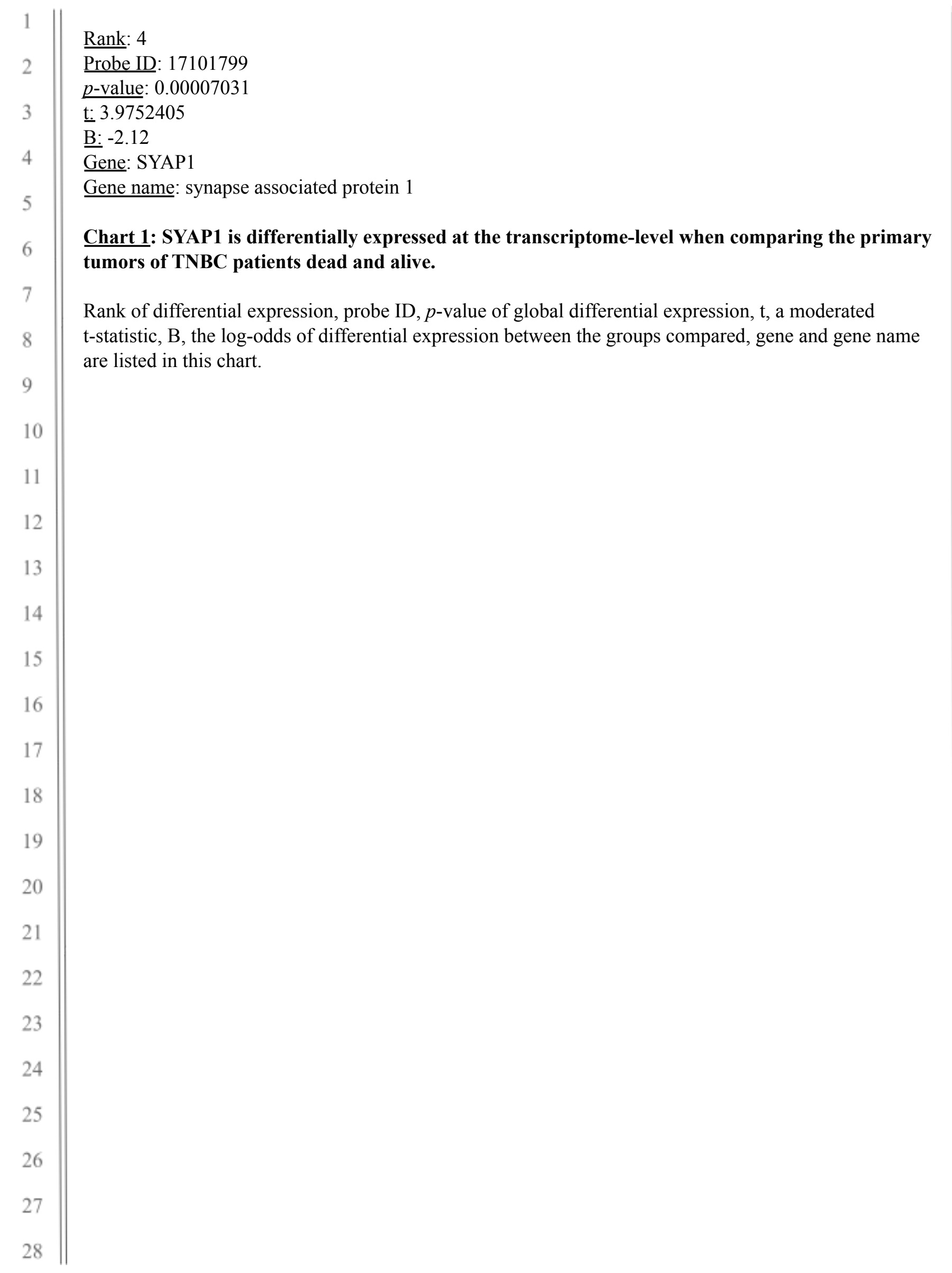




\section{SYAP1}

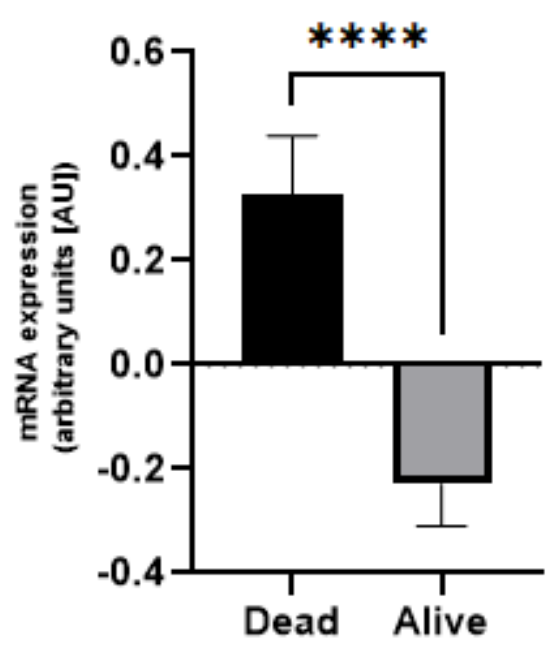

Figure 1: SYAP1 is expressed at significantly lower levels in the primary tumors of TNBC patients alive as compared to the primary tumors of TNBC patients dead.

The mRNA expression level of SYAP1 in the primary tumors of TNBC patients dead (left) and in primary tumors of TNBC patients alive (right) is graphically depicted with the result of a statistical test evaluating significance of difference in SYAP1 expression between the primary tumors of TNBC patients dead and alive, a $p$-value, listed here: $p<0.0001$. 


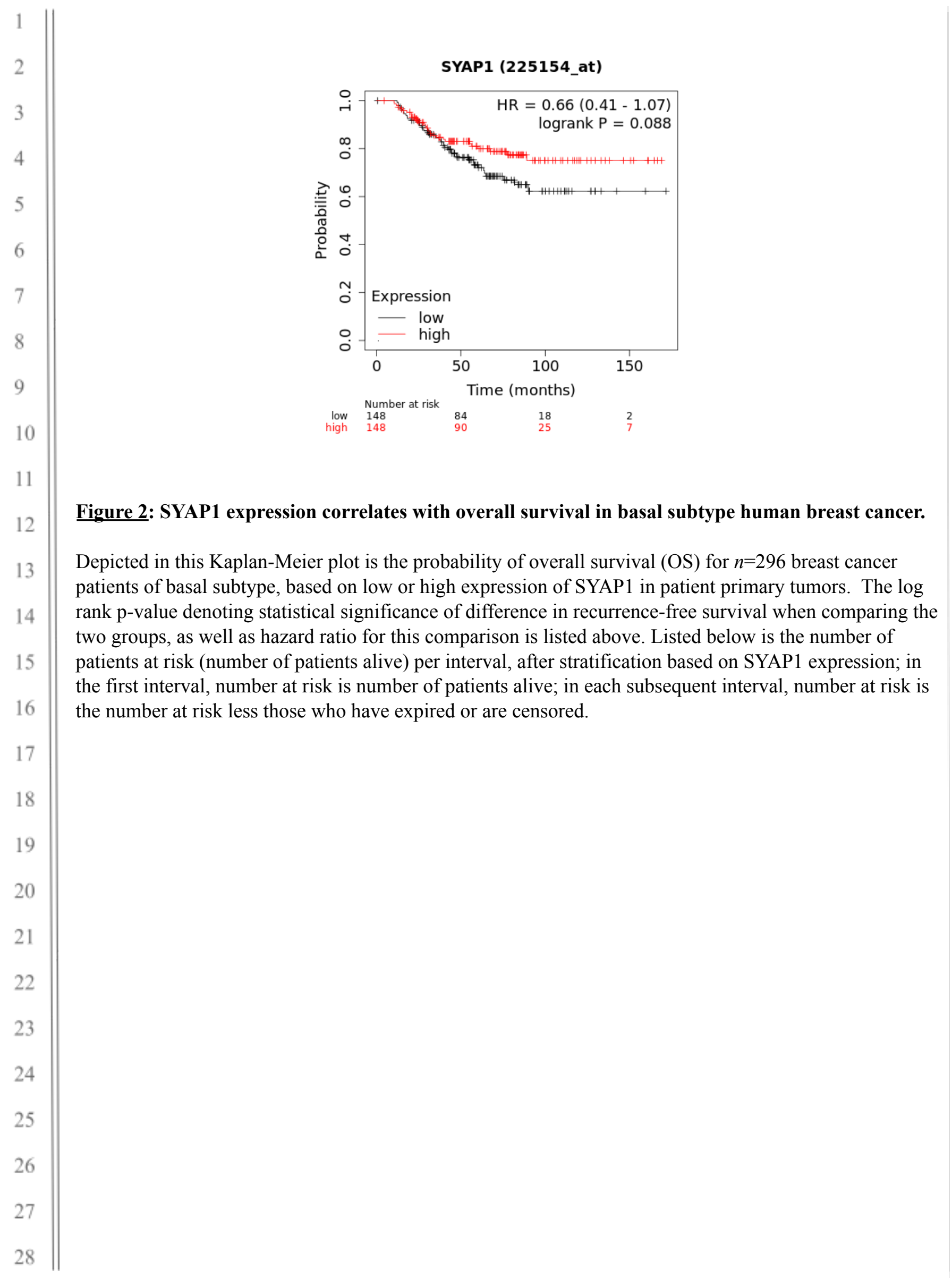

